\title{
Research on test and evaluation method of laser wireless power transmission system
}

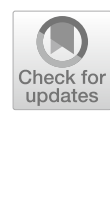

Wanli Xu${ }^{1}$, Xudong Wang ${ }^{1}$, Weishi $\mathrm{Li}^{2}$, Shizhan $\mathrm{Li}^{1}$ and Changbo Lu ${ }^{1 *}$

\section{*Correspondence:}

energylcb@163.com

${ }^{1}$ Institute of System

Engineering, Academy

of Military Science, Beijing,

China

Full list of author information

is available at the end of the

article

\begin{abstract}
Laser wireless power transmission technology avoids the traditional cable transmission requirements regarding the territory and environment, and facilitates vehicle-mounted laser wireless power transmission to provide accompanying power supplies to multitarget unmanned clusters. This can reduce the dependence of unmanned equipment (especially unmanned aerial vehicles) on power storage batteries, providing broad application prospects for the battlefield. However, the current laser wireless power transmission technology lacks a standardized technical index system and mature test and evaluation method; this is not conducive to the application of this technology in diverse combat scenarios. Accordingly, this paper proposes specific indicators for the component-level and system-level performance testing of laser wireless power transmission technology and constructs a corresponding test system and evaluation method, providing a yardstick for selecting laser wireless power transmission systems adapted to the battlefield.
\end{abstract}

Keywords: Laser wireless power transmission, Test and evaluation, System level, Component level

\section{Introduction}

Laser wireless power transmission technology has the advantages of a high power density, good power convergence, good directionality, long transmission distance, and small transmitting and receiving aperture [1,2]. These advantages allow users to keep the beam focused, even after long-distance transmission; more generally, the beam is easy to focus and has better directionality. Vehicle-mounted laser wireless power transmission has the characteristics of a concomitant moving target, miniaturization, and light weight [3], making it especially suitable for powering long-distance mobile devices, and providing good development prospects for remote power transmission [4]. Accordingly, vehicle-mounted laser wireless power transmission can be used to provide a remote power supply and communication for unmanned aircraft under navigation [5, 6]. Examples of vehicle-mounted laser wireless power transmission applications are shown in Fig. 1.

In the process of long-range wireless power transmission, the transmission efficiency index of the system is extremely important. Commonly, the system includes a laser [7], transmitting antenna, collimation system, and photovoltaic [8]. The laser performance, transmitting antenna performance [9], and other factors will directly affect the system

(c) The Author(s) 2022. Open Access This article is licensed under a Creative Commons Attribution 4.0 International License, which permits use, sharing, adaptation, distribution and reproduction in any medium or format, as long as you give appropriate credit to the original author(s) and the source, provide a link to the Creative Commons licence, and indicate if changes were made. The images or other third party material in this article are included in the article's Creative Commons licence, unless indicated otherwise in a credit line to the material. If material is not included in the article's Creative Commons licence and your intended use is not permitted by statutory regulation or exceeds the permitted use, you will need to obtain permission directly from the copyright holder. To view a copy of this licence, visit http:// creativecommons.org/licenses/by/4.0/. 


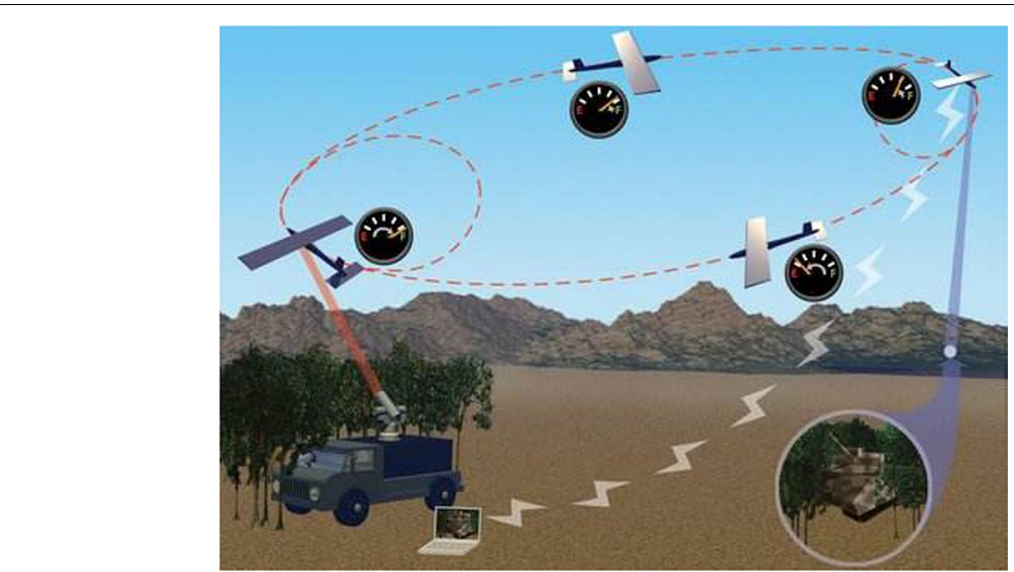

Fig. 1 Vehicle-mounted laser wireless power transmission applications

transmission efficiency [10]. It is particularly important to construct a laser wireless power transmission system test and evaluation method from both the component and overall perspectives, so as to guide the design of system components and selection of laser wireless power transmission systems adapted to the battlefield [11, 12].

This paper presents system-level and component-level tests and evaluation methods, including test conditions, test objects, test devices, test procedures, evaluation indexes, and analysis methods, aiming to provide theoretical and experimental support for the evaluation and development of laser wireless power transmission technologies [13, 14].

\section{Methods}

\subsection{Laser system performance test evaluation test}

\subsubsection{Test object}

The laser system in this study contains a laser power supply, laser, and water cooler, and the test objects are the power of the laser power supply, power after water cooling, optical power of the laser, and near-field optical power after collimation.

\subsubsection{Testing device}

The test instruments used for laser system testing include an AC power meter, DC power meter [15], laser power meter, and test device; the system arrangement is shown in Fig. 2.

\subsubsection{Test methods}

First, the test instruments are placed and connected in the order shown in Fig. 1. The focusing lens set is selected to ensure that the beam is incident from the center of the laser power meter light receiving surface. (When the laser beam diameter is too large, i.e., larger than the laser power meter probe size, we need to use the lens to focus the beam, i.e., to ensure that the measured spot is projected into the laser power meter light receiving surface diameter at a $2 / 3$ area; the judgment process is shown in Fig. 3.) The suitable optical power meter range is selected, and light gates are used block the light entering the laser power meter, to avoid instability immediately after the laser opens and damage to the instrument after a long laser exposure. 


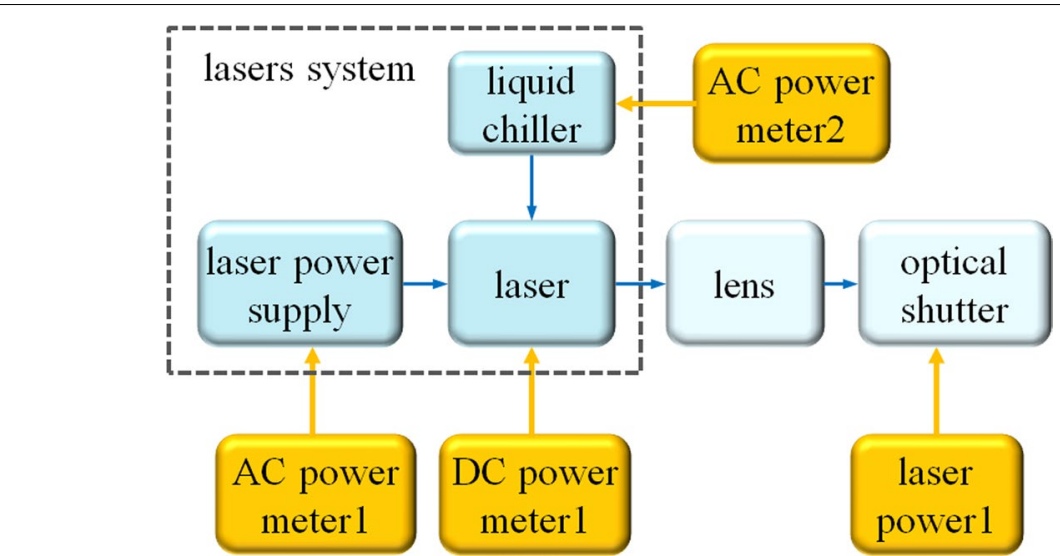

Fig. 2 Laser system test setup diagram

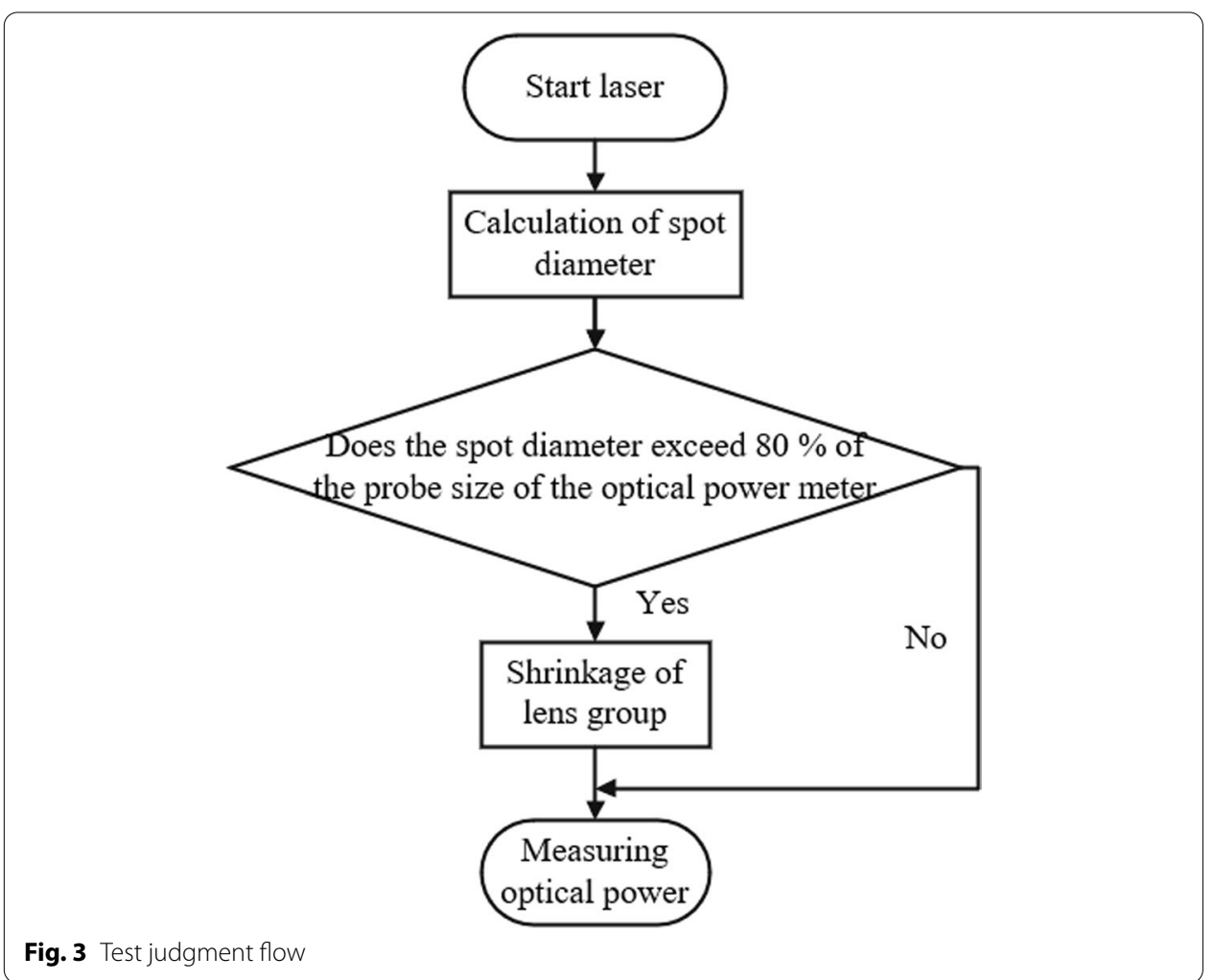

The power supply is adjusted such that the laser emits the specified power.

Two AC power meters are selected, along with DC power meters of the appropriate range. According to the specified time interval, the system records the $\mathrm{AC}$ power meter 1 reading $P_{a i}, \mathrm{AC}$ power meter 2 reading $P_{F i}$, DC power meter 1 reading $P_{d i}$, and laser power meter 1 reading $P_{i}$.

\subsubsection{Evaluation indicators}

The performance of the laser is mainly evaluated based on the efficiency of the laser, the efficiency of the laser with the laser power supply, and the efficiency of the entire system 
of the laser [16]; these need to be calculated from the measured AC power, DC power, and optical power at the transmitting end.

The AC power $P_{a 1}, P_{a 2}$, DC power $P_{d}$, and optical power $P_{\text {in }}$ at the transmitter can be calculated as follows:

$$
\begin{gathered}
P_{a 1}=\frac{1}{n} \sum_{i=1}^{n} P_{a i} \\
P_{a 2}=\frac{1}{n} \sum_{i=1}^{n} P_{F i} \\
P_{d}=\frac{1}{n} \sum_{i=1}^{n} P_{d i} \\
P_{\text {in }}=\frac{1}{n} \sum_{i=1}^{n} P_{i}
\end{gathered}
$$

In the above, $P_{a 1}$ is the input AC power in watts (W);

$P_{a 2}$ is the AC power in watts (W) passing through the water cooler;

$P_{d} \quad$ is the DC supply power of the laser in watts (W);

$P_{\text {in }}$ is the optical power in watts (W) at the transmitting end;

$n \quad$ is the number of tests;

$P_{a i} \quad P_{F i}, P_{d i}$ and $P_{i}$ are the $\mathrm{i}$-th recorded readings of each meter, respectively, in watts $(\mathrm{W})$.

The laser system evaluation contains three evaluation indexes: the efficiency of the laser $\eta_{1}$ (DC-optical power conversion efficiency), efficiency of the laser with a laser power supply (AC-optical power conversion efficiency) $\eta_{2}$, and efficiency of the laser system $\eta_{3}$.

The electrical (DC)-optical conversion efficiency, $\eta_{1}$, is calculated as follows.

$$
\eta_{1}=\frac{P_{\text {in }}}{P_{d}} \times 100 \%
$$

The electric (AC)-light conversion efficiency, $\eta_{2}$, is calculated as follows.

$$
\eta_{2}=\frac{P_{\text {in }}}{P_{a 2}} \times 100 \%
$$

The electrical-optical conversion efficiency of the laser system, $\eta_{3}$, is calculated as follows.

$$
\eta_{3}=\frac{P_{\text {in }}}{P_{a 1}} \times 100 \%
$$

\subsection{Collimation system performance test evaluation test}

\subsubsection{Test object}

The collimation system contains a collimation antenna and lens set, and the test objects are the optical power before collimation, optical power in the near and far fields after collimation, and optical power distribution map. 


\subsubsection{Testing device}

The collimation system test used in the test instruments comprises a laser power meter and spot analyzer, and the collimation system performance can be evaluated based on the light power distribution in the optical path and the spatial spot aberration situation. For the laser system device (this device is the same as that used in the Laser System Performance Test Evaluation test), the test is divided into near-field and far-field tests. If near-field beam divergence angle is small, a lens group can be added to assist in directly measuring the optical power, and an attenuator can be combined with the spot analyzer to obtain the light power distribution and spot diameter.

The test setup and system arrangement are shown in Fig. 4.

\subsubsection{Test methods}

The collimated antenna aperture $R$, distance $D$ between spot analyzers 1 and 2 , and laser cell area $S$ are measured using a ruler, and the mass $M_{1}$ of the collimated antenna is measured using a balance.

The elements are placed in the order shown in Fig. 4 and connected to the test instrument. The optical power $P_{\text {in }}$ test process at the transmitter side as measured by laser power meter 1 is the same as that described in laser system performance test evaluation test.

To select the appropriate ranges for the three laser power meters and provide a collimated near-field optical power measurement with the same test method before collimation, an appropriate focusing lens set is selected (when the laser beam diameter is too large, i.e., greater the laser power meter probe size, the lens needs to be used to focus the beam, to ensure that the measured spot is projected into the laser power meter light receiving surface diameter to $2 / 3$ of the region), so as to ensure that the beam from the laser power meter 2 light receiving surface center incidence and spot area of the collimated beam after the far-field transmission exactly and completely cover the laser cell.

The scanning range, scanning resolution, and scanning sensitivity of the spot analyzer are set according to the laser light output spectral range, and an attenuator is used for the beam before and after collimation to attenuate the light to the normal operating range of the spot analyzer.

For collimation of the far-field beam using a diaphragm, the opening is circular, and the area is denoted as $S_{o}$ (with diameter and optical power meter probe size being set to

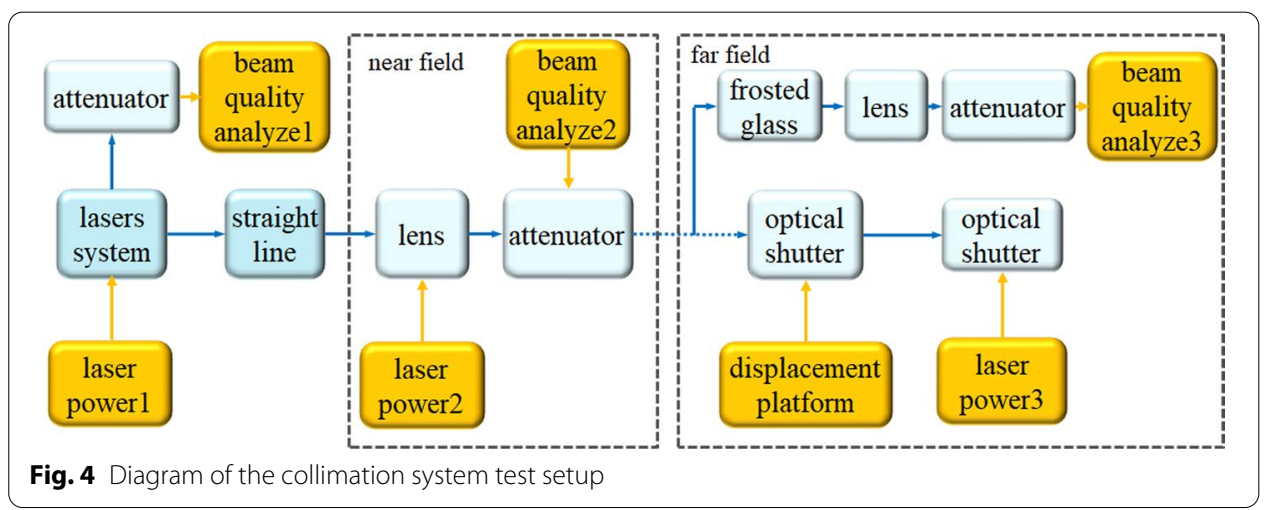


match). The diaphragm and far-field test instruments are placed on an X-Y-axis displacement table, and the diaphragm outlet is connected to the optical power meter. The farfield measurement instruments are placed a block of glass to receive the far-field large spot, with a lens to collect light into the spot analyzer.

The power supply is adjusted so that the laser emits the specified power. The spot analyzer scans in the set waveband, the spot analyzer 1 measures the beam divergence angle $\phi$, and the spot analyzer 2 measures the spot diameter $D_{1}$.

The $\mathrm{X}$-Y-axis displacement table is operated to move the light baffle in the $\mathrm{X}$ - and $\mathrm{Y}$-axis directions, and the size of the movement range is set so as to consistent with the size of the laser cell at the receiving end, allowing the user to obtain different optical power and light power distributions at the corresponding position of $x_{i} y_{j}$.

The readings $\left(P_{i}\right.$ from laser power meter $1, P_{z i}$ from laser power meter $2, P_{x_{i} y_{j}}$ from laser power meter 3 , beam divergence angle $\phi$ as measured by spot analyzer 1 , spot diameter $D_{1}$ as measured by spot analyzer 2 , and light power distribution maps as measured by spot analyzers 1,2, and 3 are recorded at specified time intervals.

\subsubsection{Evaluation indicators}

The performance of the collimation system is mainly evaluated according to collimated antenna's beam reduction ratio, near-field efficiency, optical power density at the transmitting end, and far-field spatial optical power transmission efficiency. The optical power at the transmitting end from the near- and far-field tests needs to be obtained, and the efficiency value is calculated from the spot size before and after collimation.

The optical power $P_{\text {in }}$ at the transmitter side is calculated in the same way as $P_{\text {in }}$ in laser system performance test evaluation test. The formula for calculating the near-field optical power $P_{z}$ after collimation is as follows:

$$
P_{z}=\frac{1}{n} \sum_{i=1}^{n} P_{z i}
$$

In the above,

$P_{z} \quad$ is near-field optical power in watts (W) after collimation at the emitter;

$n \quad$ is the number of tests;

$P_{z i}$ is the $\mathrm{i}$-th recorded reading of laser power meter 2 in watts (W).

The optical power $P_{o}$ received by the far-field laser cell after collimation is calculated as follows:

$$
P_{o}=\frac{\sum_{i=1}^{n} \sum_{j=1}^{n} P_{x_{i} y_{j}}}{n^{2} S_{o}} \times S
$$

The far-field cell-received optical power can be calculated as the average optical power density (the single optical power divided by the diaphragm area, and then the average value $\times$ the laser cell area).

In the above equation, $P_{o}$ is the far-field optical power in watts $(\mathrm{W})$ after collimation at the transmitter;

$n$ is the number of tests; and 
$P_{x_{i} y_{j}}$ is the recorded laser power meter reading in watts (W) from the $i$ th time in the $\mathrm{X}$-axis direction and the $\mathrm{j}$-th time in the $\mathrm{Y}$-axis direction.

The collimated antenna inlet and outlet optical power losses can be determined using the near-field power transmission efficiency.

The equation for the near-field efficiency of the collimated antenna is as follows:

$$
\eta_{4}=\frac{P_{z}}{P_{\text {in }}} \times 100 \%
$$

The equation for calculating the far-field spatial optical power transmission efficiency is as follows:

$$
\eta_{5}=\frac{P_{o}}{P_{z}} \times 100 \%
$$

The collimated antenna beam reduction capacity can be evaluated based on the ratio of the spot diameter at a certain distance without collimation to the spot diameter at the same distance after collimation; it is defined here as the collimated antenna beam reduction ratio $\beta$.

$$
\begin{aligned}
& \beta=\frac{D_{2}}{D_{1}} \\
& D_{2}=2 D \times \tan \frac{\phi}{2}
\end{aligned}
$$

Here, $D_{1}$ is the spot diameter after collimation, $D_{2}$ is the spot diameter at a certain distance without collimation, and $D$ is the distance between spot analyzers 1 and 2 .

The miniaturization and light-weighting of the collimated antennas can be determined using the optical power density at the transmitter.

The area optical power density is calculated as follows: $\rho_{1}=\frac{P_{z}}{\pi R^{2}}$.

The mass optical power density is calculated as follows: $\rho_{2}=\frac{P_{z}}{M_{1}}$.

By comparing the light power distribution maps of the near and far fields before and after collimation, the spatial spot distortion and beam uniformity can be determined, and can also be used as a reference indicator for evaluating the collimation system.

\subsection{Turntable performance evaluation test}

\subsubsection{Test object}

The test objects of the rotary table are the positioning accuracy, dynamic error, and delay error.

\subsubsection{Testing device}

The testing device comprises a self-collimating latitude and longitude meter, multifaceted prism, digital inclinometer, tripod, DC high-voltage generator, high-voltage pulse power supply, high-voltage probe, current transformer, Bayonet Neill-Concelman connector with shielded cable, and oscilloscope. 


\subsubsection{Test methods}

Rotation angle detection-The load on is placed on the rotary table and fastened; the rotation angle is set through software control to judge whether the angle of direct attainment is qualified.

Rotation speed detection-The load on is placed on the rotary table and fastened; the rotary table orientation and/or high/low axis are controlled for sinusoidal motion through software, with an amplitude of $10.67^{\circ}$ and frequency of $0.60 \mathrm{~Hz}$ (corresponding to a maximum rotation speed of $40 \% \mathrm{~s}$ and maximum rotation acceleration of $150^{\circ} / \mathrm{s}^{2}$ ). After the sinusoidal motion is stable, the actual displacement curve is plotted by collecting the feedback values of the shaft encoder. The data are compared with the theoretical displacement curve. According to the double-decade index, if the difference between the actual motion amplitude $A$ and theoretical motion displacement amplitude $|\dot{A}-A|<10 \% A$, and the difference between the actual motion phase $\dot{\phi}$ and theoretical motion phase $\phi$ satisfies $|\hat{\phi}-\phi|<10 \% \phi$, it is considered that the maximum rotation speed of the rotary table orientation or high and low axis meets the standard.

Accuracy-preserving angular acceleration detection-If the double ten index is satisfied, the accuracy-preserving angular acceleration of the rotary table orientation and/or high and low axes are considered to be up to standard.

Position accuracy testing-The turntable is leveled with the azimuth at $0^{\circ}$, and a 24-sided prism is installed in the center of rotation of the azimuth axis of the turntable. Either side of the prism is adjusted to collimate with the self-collimating latitude and longitude instrument at the observation point, and the reading of the turntable is recorded as $C_{0}$. The azimuth is rotated to $360^{\circ}$ at $15^{\circ}$ intervals in the positive direction of the azimuth, and the turntable is observed and recorded after collimation from $C_{1}$ to $C_{23}$. Then, the azimuth is rotated to $360^{\circ}$ at $15^{\circ}$ intervals in the negative direction of the azimuth, and the readings from $C_{1}$ to $C_{23}$ are observed and recorded. The data are processed in the following way $\delta=C_{i}-C_{0}$, where $\delta$ is the azimuth accuracy of each measurement position; $C_{0}$ is the value of the rotary table reading at the zero position; and $C_{i}$ is the result of the calculation of the rotary table reading at each measurement point. If the $\delta$ value is within $1.0^{\circ}$, the value is considered as passing the standard. The high- and low-axis position accuracy detection methods are also reflected in the azimuth axis method.

Dynamic error detection-If the double-ten index is satisfied, the difference between the actual motion amplitude $A$ and theoretical motion displacement amplitude $|A-A|$ is considered as the detection result.

Slip-ring performance test-The slip-ring resistance is measured with a multimeter in the ohmic range, and if it is less than $1 \Omega$, it is considered to meet the requirements. The pulse width of the high-voltage pulse power supply is set to $1 \mathrm{us}$, and the frequency is set to $150 \mathrm{~Hz}$. The high-voltage pulse power supply is connected directly to the equivalent load, and the voltage and current waveforms are measured under the actual maximum voltage and used as the reference values. The high-voltage pulse power supply is connected to the equivalent load through the high-voltage slip ring, and the voltage and current waveforms at the actual maximum voltage are measured under the static and dynamic conditions of the rotary table, to be used as the test 
values. From comparing the waveforms of the two working conditions, it can be seen that the consistency is good, there is no evident distortion, and the high-voltage process (in regard to fire discharge) is in line with the requirements.

Communication test-The network port debugging assistant is used to connect to the turntable device and send I6112 commands to the turntable controller within a fixed time period $(\mathrm{t})$. The difference in the internal timer of the controller can be obtained by subtracting the two adjacent replies, and is recorded as $\delta_{t}$. As the controller command runs for 442 us, the control delay is $\delta_{t}=0.442 \times \delta_{t}-t$, in ms; the average value after several measurements is taken as the final measurement result.

\subsubsection{Evaluation indicators}

The performance of the rotary table is evaluated in terms of the equipment integrity, directional axis positioning accuracy, and dynamic errors.

\subsection{Laser cell component performance test evaluation test}

\subsubsection{Test object}

The laser cell assembly contains the laser cell, maximum power tracking module $[17,18]$, voltage regulator module, and load. The test objects are the optical power at the laser cell end, and the electrical power output of each module [19].

\subsubsection{Testing device}

The test instruments used for the laser cell component testing are a laser power meter, voltammetric characteristic tester, and DC power meter, and the auxiliary instruments are a 3D displacement table and temperature controller, to realize control of the angle and temperature.

The static test setup and system placement are shown in Fig. 5.

\subsubsection{Test methods}

The laser cell area $S$ and mass $M_{2}$ are measured.

The test apparatus is placed and connected with the parts in the order shown in Fig. 5, with the laser cell placed on a three-dimensional displacement table with an adjustable angle and a temperature controller for regulating the temperature at the cell end.

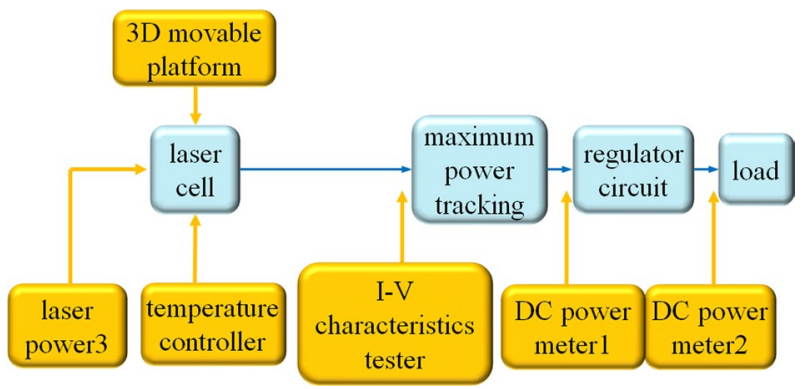

Fig. 5 Receiver side battery component test setup diagram—static 
The laser power test is conducted with reference to far-field optical power measurement method described in collimation system performance test evaluation test; here, the far-field optical power $P_{o}$ denotes the laser cell's received optical power.

The laser power supply is adjusted so that the laser emits the specified power, and the turntable at the transmitting end automatically adjusts the position of the emitted laser to ensure that the beam is incident from the center of the receiving surface of the laser cell.

The appropriate range is selected for the voltammetric tester. First, the voltammetric tester is used to connect the laser cell alone, so as to measure the maximum power point $P_{m i}$ of the laser cell, as well as other parameters (see the data log sheet for details).

The three-dimensional displacement table is adjusted so that the light beam is incident on the battery module at a certain angle $\theta$. The temperature controller controls the temperature of the battery module to a certain value, selects the appropriate range for the two DC power meters, and records the readings $P_{d o 1 i}$ of DC power meter 1 and $P_{d o 2 i}$ of DC power meter 2 according to the specified time interval.

The three-dimensional displacement table is adjusted to change the laser incidence angle $\theta$ and the temperature is kept constant; then, the readings $P_{d o 1 i}$ of DC power meter 1 and $P_{d o 2 i}$ of DC power meter 2 are recorded at the specified time intervals.

The temperature of the battery component is changed, the laser incidence angle $\theta$ is kept constant, and the readings $P_{d o 1 i}$ of DC power meter 1 and $P_{d o 2 i}$ of DC power meter 2 are recorded at the specified time intervals.

The laser emission power is changed, the laser incidence angle $\theta$ is kept constant, the temperature is kept constant, and $P_{m i}, P_{d o 1 i}$, and $P_{d o 2 i}$ are recorded at prescribed time intervals (here, the optical power gradient is set to strong light and weak light).

Because the dynamic system cannot measure the optical power at the receiving end, it can only ensure that in the wind speed case, the circuit parameters are measured at the receiving end, using a test device as shown in Fig. 6. The wind speed check tester monitors the wind speed flowing through the heat sink, and the optical power is adjusted at the transmitting end. The voltammetric characteristics tester readings Pmi and other parameters are recorded (see data recording table for details), as well as the DC power meter 1 reading $P_{d o 1 i}$, and DC power meter 2 reading $P_{d o 2 i}$.

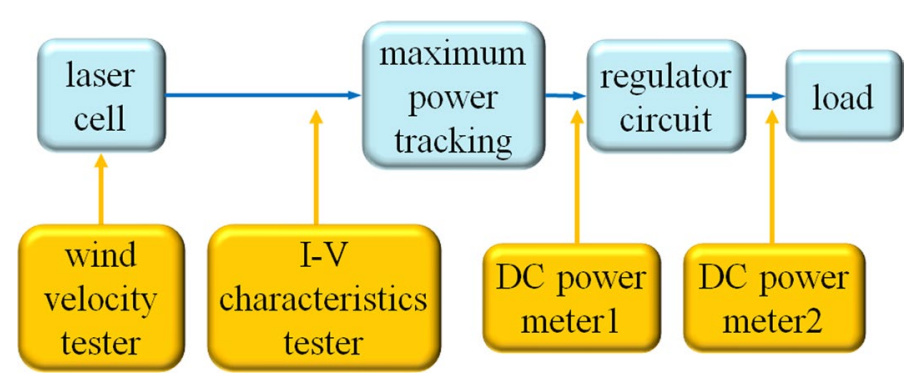

Fig. 6 Receiver side battery component test set-up diagram—dynamic 


\subsubsection{Evaluation indicators}

The performance of the cell module is mainly evaluated from the perspectives of the cell photoelectric conversion efficiency, efficiency of the maximum power tracking module, efficiency of the voltage regulator module at the receiving end, etc. It is necessary to obtain the optical power at the laser cell end and electrical power output of each module to calculate the efficiency value.

The DC power at the receiving end can be calculated for both the dynamic and static systems as follows:

$$
P_{m}=\frac{1}{n} \sum_{i=1}^{n} P_{m i} ; \quad P_{d o 1}=\frac{1}{n} \sum_{i=1}^{n} P_{d o 1 i} ; \quad P_{d o 2}=\frac{1}{n} \sum_{i=1}^{n} P_{d o 2 i}
$$

In the above,

$P_{m} \quad$ is the maximum power output of the battery in watts (W);

$P_{d o 1}$ is actual output DC power of the maximum power tracking module in watts (W);

$P_{d o 2}$ is the regulated output DC power in watts (W);

$n \quad$ is the number of tests;

$P_{m i} \quad$ is the maximum power point reading of the battery output as recorded by the voltammetric characteristic tester for the ith time in watts (W);

$P_{d o 1 i}$ is the ith recorded reading of DC power meter 1 in watts (W); and

$P_{d o 2 i}$ is the ith recorded reading of DC power meter 2 in watts (W).

For static systems, the respective corresponding efficiencies can be calculated for different angles and temperature conditions. The laser cell evaluation can refer to its photoelectric conversion efficiency $\eta_{i j}$, which is calculated as follows.

$$
\eta_{i j}=\frac{P_{m}}{P_{o}} \times 100 \%
$$

For the dynamic system, the evaluation of the influences of different angles and temperature conditions on the cell efficiency can be referred to as the average efficiency $\bar{\eta}$ for the photoelectric conversion efficiency $\eta_{i j}$ calculated for the different angle conditions $i$ and different temperature conditions $j$. The corresponding weighting factor $X_{i j}$ is added to it, the size of which is determined according to the actual needs, as follows:

$$
X_{i j}=\left[\begin{array}{ccc}
X_{11} & \cdots & X_{1 j} \\
\vdots & \ddots & \vdots \\
X_{i 1} & \cdots & X_{i j}
\end{array}\right] \quad \sum_{i=1}^{n} \sum_{j=1}^{n} X_{i j}=1
$$

The corresponding calculation of the average efficiency is as follows:

$$
\bar{\eta}=\sum X_{i j} \times \eta_{i j}
$$

To examine the effects of different laser intensities on the photoelectric conversion efficiency of the cell module, the optical power at the emitter side $P_{o}$ can be compared for different cases of $\bar{\eta}$.

The optical power density is calculated as follows: 


$$
\rho_{3}=\frac{P_{o}}{S} ; \rho_{4}=\frac{P_{o}}{M_{2}}
$$

The efficiency of the maximum power tracking module $\eta_{m}$ is calculated as follows:

$$
\eta_{m}=\frac{P_{d o 1}}{P_{m}} \times 100 \%
$$

The receiver-side voltage regulator module efficiency $\eta_{d}$ is calculated as follows:

$$
\eta_{d}=\frac{P_{d o 2}}{P_{d o 1}} \times 100 \%
$$

\subsection{Turntable performance evaluation test}

\subsubsection{Test object}

The heat dissipation system is tested based on the temperatures of the photosensitive and backlight sides of the laser cell, and the temperature after heat dissipation.

\subsubsection{Testing device}

The test instruments for the static system are an optical power meter, temperature sensor, and static system-set speed control fan to provide a fixed wind speed; for the dynamic system, the wind speed tester is set to measure the wind speed during flight, along with the test device and system placement as shown in Fig. 7.

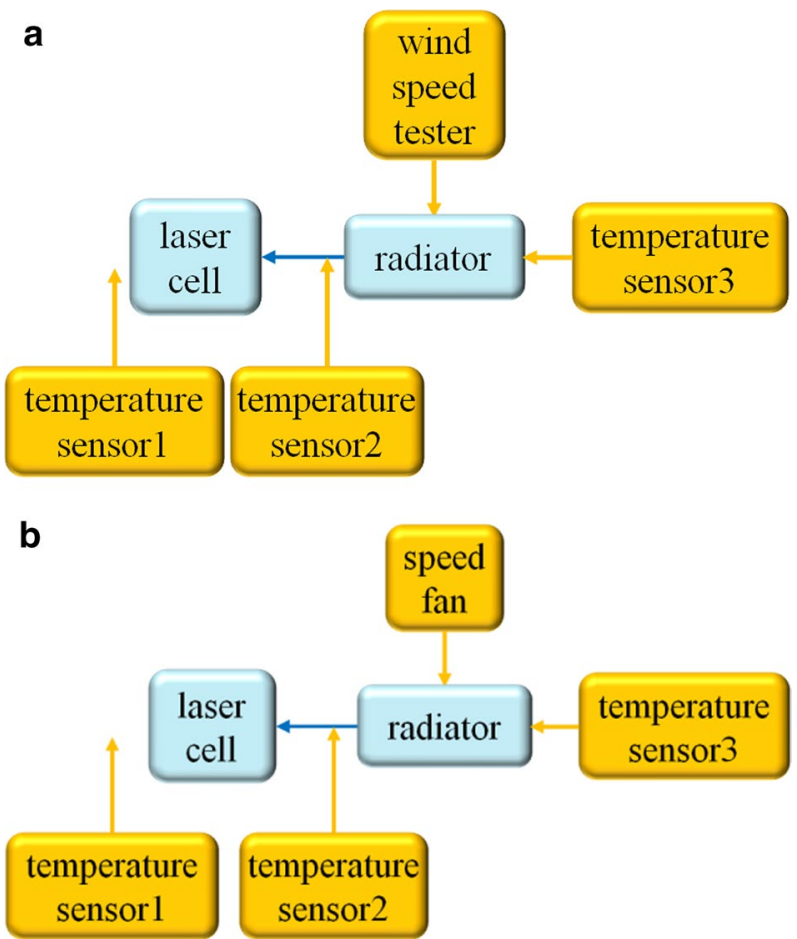

Fig. 7 Battery pack test set diagram 


\subsubsection{Test methods}

The mass $M_{3}$ of the heat sink is measured with a balance.

The elements are placed in the order shown in Fig. 7 and connected to the test instrument (for dynamic and static systems, the receiving end is a self-contained heat sink).

Temperature sensor 1 is mounted on the photosensitive surface of the battery plate, temperature sensor 2 is mounted on the back of the battery plate, and temperature sensor 3 is mounted on the heat sink of the battery plate.

The laser power supply is adjusted so that the laser emits the specified power, and the turntable at the transmitting end automatically adjusts the position of the emitted laser to ensure that the beam is incident from the center of the receiving surface of the laser cell.

For static systems, the speed-controlled fan provides a fixed air speed to the heat sink, and records the current moment air speed; for dynamic systems, only the air speed at the current moment is recorded.

The laser power $P_{z}$ at the transmitter is recorded, along with the corresponding steadystate temperature readings $T_{s 1}, T_{s 2}$, and $T_{s 3}$ in the temperature profiles obtained from the three temperature sensors.

The fan output is changed and the wind speed is recorded, along with the laser power $P_{z}$ at the transmitting end and the reading of each temperature sensor at the current moment.

The temperature change curve of the battery as measured using the temperature sensor is shown in Fig. 8.

In Fig. $8, T_{s}$ is the steady-state temperature in degrees Celsius $\left({ }^{\circ} \mathrm{C}\right)$.

\subsubsection{Evaluation indicators}

The performance of the heat dissipation system is mainly evaluated based on the temperature density, and it is necessary to obtain the temperatures $T_{s 1}, T_{s 2}$, and $T_{s 3}$ as calculated from the battery photosensitive surface, backlight surface, and after heat dissipation to obtain the temperature density. From the steady-state temperature $T_{s}$ in the temperature

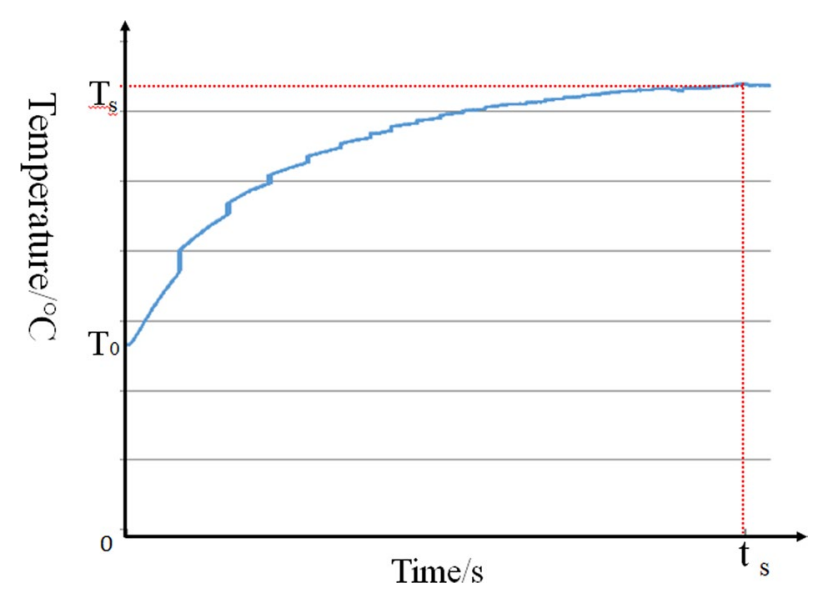

Fig. 8 Schematic diagram of laser cell temperature profile 
rise curve, the front and back sides of the battery components and heat dissipation system can be evaluated under different wind speed conditions.

For the evaluation of the lightness of the heat sink, the temperature density is $\rho_{5}=\frac{T_{s 2}-T_{s 3}}{M_{3}}$.

\section{System performance test evaluation}

\subsection{Test subjects}

The test object includes a static test object and dynamic test object, and consists of a laser power supply, laser, water chiller, collimated antenna, turntable, laser battery, heat sink, maximum power tracking module, voltage stabilization module, and load [20]. The static test object refers to a ground power transmission system with a fixed receiver end. The dynamic test object refers to a power transmission system with a movable receiver end in the process of power transmission, including but not limited to unmanned vehicles (including aerial vehicles) and robot laser wireless power transmission systems [21-23].

For the lasers, the power ranges of $0-500 \mathrm{~W}, 500-1000 \mathrm{~W}$, and $1000 \mathrm{~W}$ and above are used to facilitate the horizontal evaluation of system performance; for the power transmission distance, the distance ranges of 0-200 m, 200-1000 m, and $1000 \mathrm{~m}$ and above are used to facilitate the vertical evaluation of the near-field and far-field system performance $[24,25]$.

For static systems, the laser cell is placed on the angle-adjustable three-dimensional displacement table, with the temperature controller to control the temperature (when applicable) and the speed-controlled fan to provide the specified air speed to the heat sink (when applicable); for dynamic systems, the receiving end is required to hover at a specified speed, and on a specified path [26, 27].

\subsection{Test setup}

The overall layout of the test device is shown in Fig. 9, and mainly includes the power transmission system and test unit. The power transmission system includes the laser transmitter and receiver. The transmitter includes a laser power supply, laser, water chiller, collimated antenna, and turntable, and the receiver includes a laser battery, maximum power tracking module, voltage regulator module, and load. The test unit includes

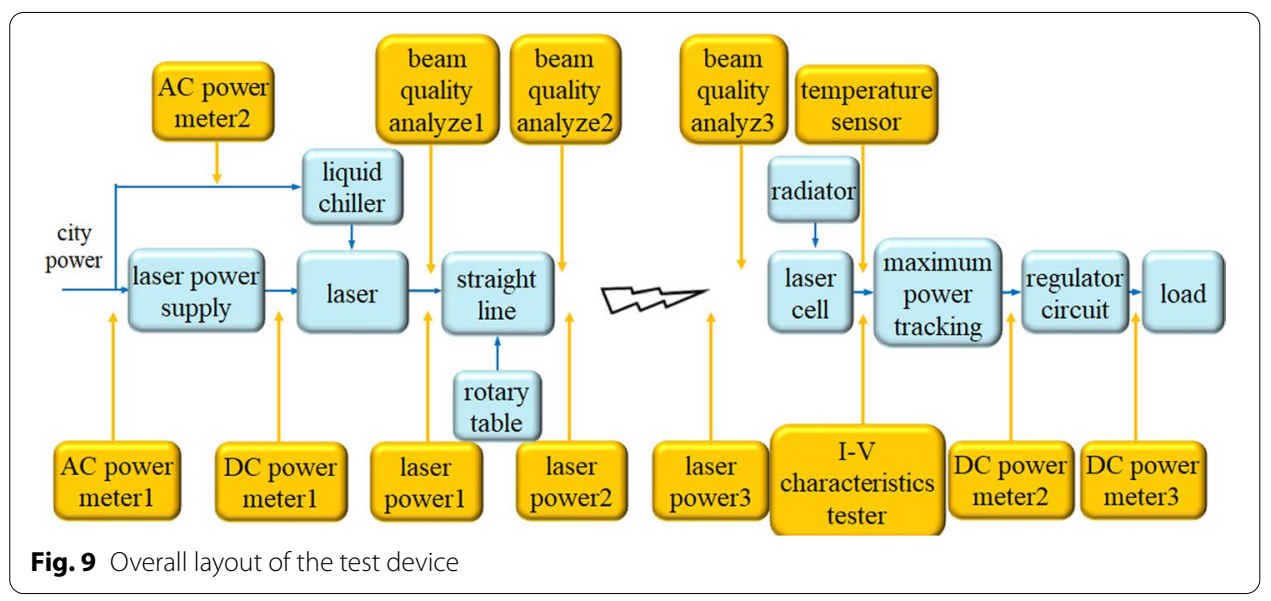


the AC power meter 1, AC power meter 2, DC power meter 1, DC Power meter 2, DC power meter 3, laser power meter 1, laser power meter 2, voltammetric characteristics tester, spectral analyzer, spot analyzer, laser rangefinder, and temperature sensor (when applicable). The more detailed test steps use an attenuator, light gate(s), temperature controller, three-dimensional displacement table, speed control fan, and other auxiliary measurement tools.

\subsection{Test methods}

\subsubsection{Site layout}

The overall layout of the site refers to the regional division of the test site to ensure the safety of test personnel and test instruments, i.e., dividing the site into a safety zone, non-safety zone, danger zone, and laser power transmission test area, as shown in Fig. 10. The safety zone personnel can observe, shoot, record, and manipulate the monitoring instrument to trigger the switch; the non-safety zone is the location where the laser may be irradiated, and no one is allowed to walk in the area; the danger zone is the area around the receiving laser battery and load, where the laser has pointing instability and the optical path may have a small deviation (again, no one can stay here). The test area is for the laser wireless power transmission system work, and is mainly used for placing the laser transmitting system (laser power supply, laser, and chiller), collimation system (transmitting antenna, lens set, and turntable), receiving system (laser battery, voltage regulator module, and load), and test instruments (AC power meter, DC power

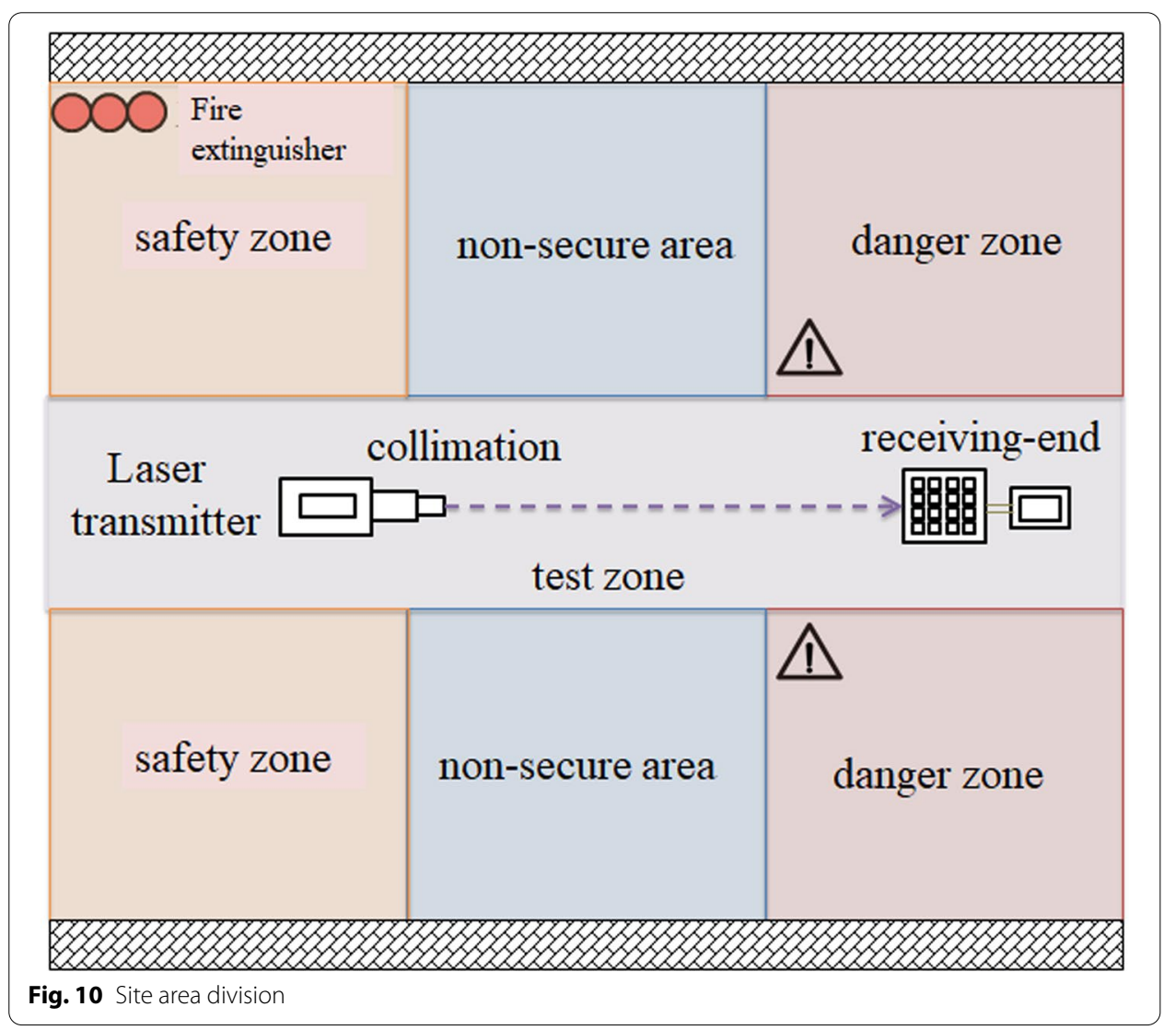


meter, digital source meter, laser power meter, voltammetric characteristic tester, spectrum analyzer, spot analyzer, laser rangefinder, and temperature sensor).

\subsection{Test setup}

\subsubsection{Measurement point arrangement}

The data measured at the component level remain valid for system performance evaluation tests, and the overall measurement point map is as follows (Fig. 11).

\subsection{Evaluation indicators}

The evaluation of the power transmission system can be calculated from the above measured parameters to calculate the overall electric-electric (DC-DC) conversion efficiency $\eta$ of the system, as follows [28].

$$
\eta=\frac{P_{d o 2}}{P_{d}} \times 100 \%
$$

\section{Conclusion}

In this study, a laser wireless power transmission technology index system is examined, and the system-level and component-level evaluation methods are clarified. The main conclusions are as follows.

(1) Component-level testing was performed on the laser system, collimation system, turntable, laser battery components, and heat dissipation system from multiple angles.

(2) System-level testing specified the test objects, test devices, test methods, and evaluation indicators, forming a complete system of technical indicators and test evaluation methods.

The proposed test method is highly operable and universal, and can be applied to the overall evaluation of the system, as well as individual evaluations of each component. It provides reliable technical support and a theoretical basis for performance evaluations of different laser wireless power transmission systems in the future. Moreover, it

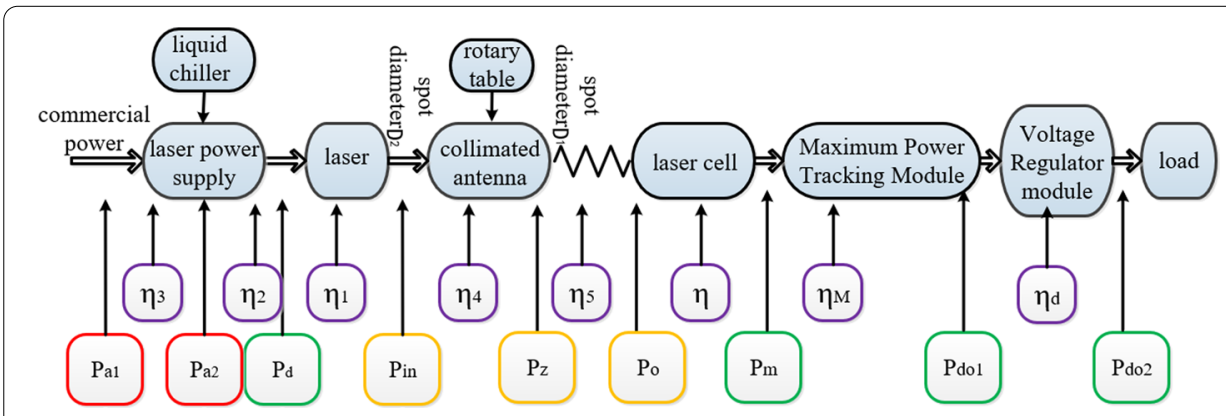

Fig. 11 Overall measurement point diagram 
provides guiding significance for the selection of laser wireless power transmission systems adapted to the battlefield.

\section{Acknowledgements}

We are grateful to the anonymous reviewers who have contributed to the enhancement of the paper's completeness with their valuable suggestions.

\section{Authors' contributions}

This paper is completed with the cooperation of five authors. WLX proposed the research direction. XDW proposed the research methods. WSL analyzed the data and wrote this manuscript. SZL carried out the translation work. CBL proofread the paper. All authors read and approved the final manuscript.

\section{Funding}

None

Availability of data and materials

The datasets used and/or analyzed during the current study are available from the corresponding author on reasonable request.

\section{Declarations}

Ethics approval and consent to participate

Not applicable.

\section{Consent for publication}

We agree to the publication of the paper.

\section{Competing interests}

The authors declare that they have no competing interests.

\section{Author details}

${ }^{1}$ Institute of System Engineering, Academy of Military Science, Beijing, China. ${ }^{2}$ Linfen Representative Office of Beijing Representative Office, Shanxi, China.

Received: 28 December 2021 Accepted: 16 February 2022

Published online: 07 March 2022

\section{References}

1. Y. Li, Q. Zhang, C. Zhang, Research on laser wireless power transmission technology for unmanned aircraft. Fly. Missile $\mathbf{0 0 0}(001), 58-61(2015)$

2. Z. Wu, J. Xie, Y. Yang, Design and implementation of beam shaping for high power semiconductor lasers. Laser Technol. 41(3), 416-420 (2017)

3. M. Alotaibi, Low noise moving target detection in high resolution radar using binary codes. EURASIP J. Adv. Signal Process. 8, 1-19(2021)

4. X. Liu, W. Hua, X. Liu, Experimental study on the output characteristics of laser irradiated monocrystalline silicon photovoltaic cells. China Laser 8,66-71 (2015)

5. M. Yan, H. Yuan, X. Jie, Task allocation and route planning of multiple UAVs in a marine environment based on an improved particle swarm optimization algorithm. EURASIP J. Adv. Signal Process. 2021, 19 (2021)

6. T. Zhou, K. Xu, X. Wei, Aerial intelligent reflecting surface-enhanced cell-free massive mimo for high-mobility communication: joint doppler compensation and power optimization. EURASIP J. Adv. Signal Process. 2021, 19 (2021)

7. L. Juan, Y. Hao, T. Yu, Design of high efficiency diode laser module for wireless power transmission. Infrared Laser Eng. 50(5), 8 (2021)

8. L. Wei, W. Lingyuan, W. Wang, Research progress of laser wireless power transmission. Laser Optoelectron. Prog. 55(2), $10(2018)$

9. L. Guo, T. Chun, D. Wu, Measurement of "smile" for high-power diode laser array. High Power Laser Part. Beams 02 , 37-40 (2009)

10. L. Qiao, Y. Yang, Experimental research of laser wireless power transmission efficiency. Laser Technol. 38(005), 590-594 (2014)

11. C. Zhao, Y. Wang, L. Guo, Development of laser wireless power transmission technology. Laser Technol. 44(5), 8 (2020)

12. L. Tang, Y. Zhou, C. Zhang, Research situation and development trend of laser wireless power transmission key technology. Laser J. 38(10), 5 (2017)

13. C. Tao, D. Zhang, K. Liu, Research on the background spectral power matching of multi-wavelength laser wireless power transmission system. Optoelectron. Technol. 41(2), 6 (2021)

14. C. Tao, D. Zhang, Y. Zhou, Experimental research on the application of laser wireless energy transmission storage energy. Laser J. 42(7), 5 (2021)

15. Test methods of semiconductor lasers

16. General specification for semiconductor lasers 
17. W. Zhongqiang, C. Bilian, L. Hou, Maximum power point tracking for photovoltaic system based on improved multiverse optimization. J. Electron. Inf. Technol. 44, 1-8 (2022)

18. K. Liu, D. Zhang, C. Tao, Multi-wavelength laser wireless energy transmission power matching and rear-stage energy storage problems. Laser J. $\mathbf{4 2}(10), 5$ (2021)

19. L. Sun, Researches on measuring model of solar cell's performance parameters. PhD thesis, Hangzhou Dianzi University (2012)

20. J. Gu, Research on compensation method of atmospheric loss in laser energy transmission. PhD thesis, JiLin University (2019)

21. X. Liu, X. Zhang, Noma-based resource allocation for cluster-based cognitive industrial internet of things. IEEE Trans. Ind. Inform. 16(8), 5379-5388 (2020)

22. X. Liu, X. Zhai, W. Lu, Qos-guarantee resource allocation for multibeam satellite industrial internet of things with noma. IEEE Trans. Ind. Inform. PP(99), 1 (2019)

23. X. Liu, X. Zhang, Rate and energy efficiency improvements for $5 \mathrm{~g}$-based iot with simultaneous transfer. IEEE Internet of Things J. PP(99), 1 (2018)

24. X. Liu, X. Zhang, M. Jia, 5g-based green broadband communication system design with simultaneous wireless information and power transfer. Phys. Commun. 28, 130-137 (2018)

25. M. Wang, Y. Lin, Q. Tian, Transfer learning promotes $6 \mathrm{~g}$ wireless communications: recent advances and future challenges. IEEE Trans. Reliab. PP(99), 1-18 (2021)

26. Y. Lin, Y. Tu, Z. Dou, Contour stella image and deep learning for signal recognition in the physical layer. IEEE Trans. Cogn. Commun. Netw. PP(99), 1 (2020)

27. Y.Tu, Y. Lin, H. Zha, Large-scale real-world radio signal recognition with deep learning (2021)

28. Terminology for Laser

\section{Publisher's note}

Springer Nature remains neutral with regard to jurisdictional claims in published maps and institutional affiliations.

\section{Submit your manuscript to a SpringerOpen ${ }^{\circ}$ journal and benefit from:}

- Convenient online submission

- Rigorous peer review

- Open access: articles freely available online

- High visibility within the field

Retaining the copyright to your article

Submit your next manuscript at $\gg$ springeropen.com 